\title{
Nuchal translucency and lymphatic system maldevelopment
}

\author{
Carlo Bellini ${ }^{1, a, *}$, Mariangela Rutigliani ${ }^{2, a}$, \\ Francesco M. Boccardo ${ }^{3}$, Eugenio Bonioli', \\ Corradino Campisi ${ }^{3}$, Federica Grillo², \\ Tommaso Bellini', Mario Valenzano ${ }^{4}$ \\ and Ezio Fulcheri ${ }^{2}$ \\ 1 Department of Pediatrics (DIPE), University of Genoa, \\ Gaslini Institute, largo G. Gaslini, 5, 16147 Genova, \\ Italy \\ 2 Department of Pathology (DICMI), S. Martino Hospital, \\ University of Genoa, viale Benedetto XV, 16145 \\ Genova, Italy \\ ${ }^{3}$ Department of Surgery, Lymphatic Surgery and \\ Microsurgery Unit, S. Martino Hospital, University of \\ Genoa, viale Benedetto XV, 16145 Genova, Italy \\ ${ }^{4}$ Department of Obstetrics and Gynecology, University \\ of Genoa, Largo R. Benzi 10, 16132 Genova, Italy
}

\begin{abstract}
We describe the histological examination of 18 aborted fetuses that had increased nuchal translucency (NT) between $11^{+0}$ and $13^{+6}$ weeks' gestation. The aim of this study was to assess the corresponding NT anatomic features by immunohistochemical (IHC) investigation. A morphological study was performed using lymphatic and blood endothelial specific markers, as well as smooth muscle actin (SMA). We found that all 18 cases were D2-40 positive, CD31 positive, and CD34 negative, suggesting the presence of nuchal lymph vessel ectasia. We found that $12 / 18$ cases were SMA staining positive and $6 / 18$ cases were SMA negative, suggesting that 6/18 cases had nuchal cystic lymphangiectasia, whereas $12 / 18$ had cystic hygromas. The present data seem to confirm the reasonable hypothesis that lymphangiogenesis plays a relevant role in nuchal edema, increased NT, and that increased NT is the result of a lymphatic malformation or a delayed development of the lymphatic system.
\end{abstract}

Keywords: Cystic hygroma; edema with nuchal cystic lymphangiectasia; nuchal translucency (NT).

\footnotetext{
aBoth authors contributed equally to the paper.

${ }^{*}$ Corresponding author:

Carlo Bellini, MD, PhD

Dipartimento di Pediatria

Università di Genova

Istituto G. Gaslini, Largo G. Gaslini 5

16147 Genova

Italy

Tel.: +39105636762

Fax: + 39103770675

E-mail: carlobellini@ospedale-gaslini.ge.it
}

\section{Introduction}

Fetal nuchal translucency (NT) is a small translucent area in the posterior fetal neck [18] which can be visualized by ultrasound examination between the $11^{+0}$ and $13^{+6}$ weeks of gestation (Fetal Medicine Foundation, London, UK: http://www.fetalmedicine.com/). NT usually disappears after 14 weeks of gestation [15]. Increased NT is defined as $>95^{\text {th }}$ percentile and represents edema behind the fetal neck. It has been established that measuring NT between the $11^{+0}$ and $13^{+6}$ weeks' gestation, together with maternal age and serum biochemistry is a sensitive, accurate and effective method of first-trimester screening for chromosomal abnormalities [14, 19]. The higher the NT, the greater the probability of aneuploidy, cardiac defects, anomalies, and genetic syndromes. Although NT measurement is performed worldwide and a commonly used screening method, its pathophysiology is still unclear $[4,8,9,12]$. This paper describes a retrospective histological examination of 18 aborted fetuses that had increased NT between the $11^{+0}$ and $13^{+6}$ weeks' gestation. The aim of this study was to relate the NT anatomic features to immunohistochemical findings.

\section{Materials and methods}

This was a retrospective cohort study reviewing 1098 autopsy files. Figure 1 shows the subject inclusion-exclusion flow-chart. All fetuses were prenatally examined by ultrasound and diagnosed as normal or increased NT measurement performed between the $11^{+0}$ and $13^{+6}$ weeks' gestation. The fetuses were karyotyped by chorionic villus sampling or amniocentesis. Termination of the pregnancy was performed at the request of the parents for medical or psychosocial reasons, according to the current Italian law (Law 194/1978). The medical Ethical Committee approved the study. All parents received information and gave written informed consent.

Autopsies of retrieved-scheduled cases were performed according to a previously described protocol [11]. Consecutive parallel sections were obtained cutting along cross-sectional planes. Of all the sections, the one running from the mental spine, at the lower jawbone margin, to the third cervical vertebra was the reference section to fully examine the nuchal region.

Five $\mu$ thick microtome sections were obtained from paraffin blocks used for routine hematoxylin and eosin staining and for immunohistochemical (IHC) investigations. The following monoclonal antibodies were used: CD31 antibodies (Dako, Glostrup, Denmark, 1A10), CD34 (Dako, Glostrup, Denmark, QBEnd10) [17], D2-40 (Signet, England, D2-40), smooth muscle actin (SMA) (Ventana, Arizona, USA, 1A4) [15, 16]. D2-40 was diluted $1: 120$ and incubated for $60 \mathrm{~min}$ at room temperature. Antigenic 


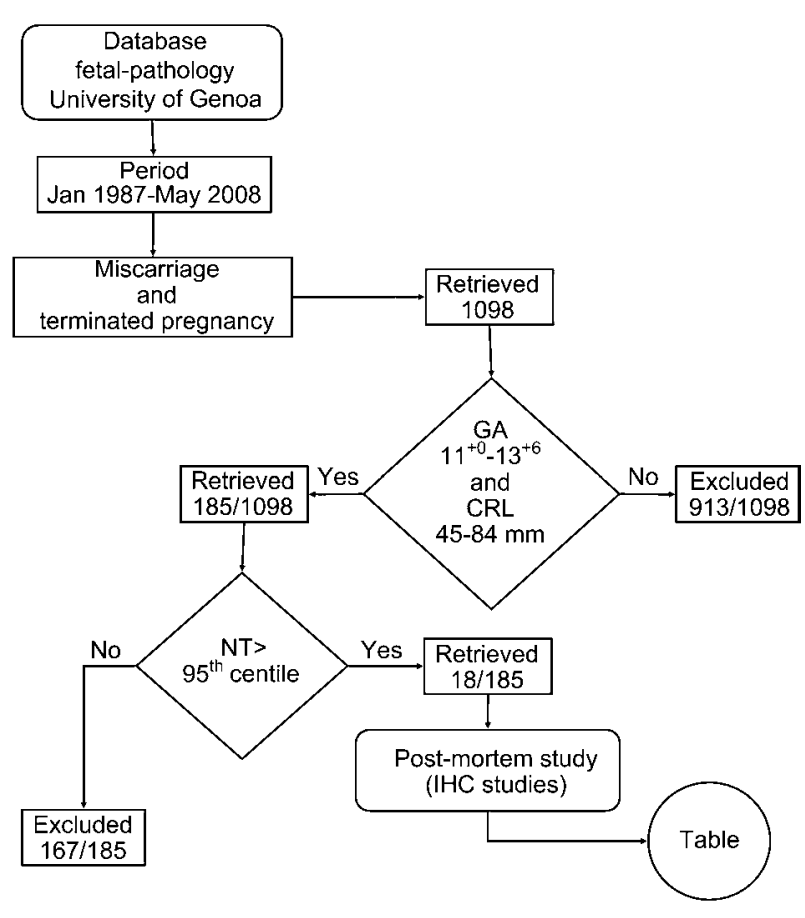

Figure 1 Subject inclusion-exclusion flow-chart. $\mathrm{GA}=$ gestational age, $\mathrm{CRL}=$ crown-rump length, $\mathrm{NT}=$ nuchal translucency, $\mathrm{IHC}=$ immunohistochemical.

unmasking was carried out using subsequent microwave cycles (each cycle takes from 4 to $32 \mathrm{~min}$ at $37^{\circ} \mathrm{C}$ ) except for SMA. A Ventana kit was employed for endogenous Biotin block.

CD31 is platelet endothelial cell adhesion molecule-1 (PECAM-1) that is highly specific for lymphatic and bloodvascular endothelium [16]. CD34 is expressed by embryonic cells of the hematopoietic system, including endothelial cells that are present in blood-vascular vessels [17]. SMA antibody is useful for identifying smooth muscle cells of the bronchial and bronchiolar wall as well as of vessel wall. It can stain myofibroblasts and muscle cells in the intima and in the media of small and medium caliber lymph vessels $[6,16,17]$. Podoplanin is a transmembrane mucoprotein that is recognized by the D2-40 monoclonal antibody. D2-40 is a selective monoclonal immunohistochemical marker of lymphatic endothelium on formalinfixed and paraffin-embedded tissue specimens [10].

\section{Results}

We identified 18 fetuses that matched our inclusion criteria (Figure 1). The main characteristics of the fetuses and the IHC results are summarized in Table 1. Results of the histological examination related to the nuchal region as performed by H\&E staining in tissue samples from all 18 enrolled fetuses suggested the presence of lymph vessel lesions at the nuchal level (Figure 2, panels A1 and A2). IHC studies demonstrated that the endothelial profile along optically empty spaces at the nuchal level were D2-40 positive, CD31 positive, and CD34 negative in all 18 enrolled fetuses (Figure 2, panels B1 and B2). SMA staining was highly positive in $12 / 18$ cases and practically absent (i.e., negative) in the other 6/18 cases (Figure 2, panels C1 and C2; Table 1).

\section{Discussion}

We used IHC methods to examine the nuchal lymphatic system of 18 aborted fetuses with increased NT meas-

Table 1 Immunohistochemical study results in 18 fetuses with increased fetal nuchal translucency between the $11^{+0}$ and $13^{+6}$ weeks' gestation.

\begin{tabular}{|c|c|c|c|c|c|c|c|c|c|}
\hline & & \multirow[t]{2}{*}{ GA } & \multirow[t]{2}{*}{ Karyotype } & \multirow{2}{*}{$\begin{array}{l}\text { Associated conditions } \\
\text { and malformations }\end{array}$} & \multicolumn{4}{|c|}{ Immunohistochemical staining } & \multirow{2}{*}{$\begin{array}{l}\text { NT } \\
\text { anatomical } \\
\text { equivalent }\end{array}$} \\
\hline & & & & & CD31 & CD34 & $\mathrm{D} 2-40$ & SMA & \\
\hline 1 & TOP & $11+0$ & $46, X Y$ & Normal & POS & NEG & POS & NEG & $\mathrm{CL}$ \\
\hline 2 & TOP & $12+4$ & $46, X X$ & Hydrops & POS & NEG & POS & NEG & $\mathrm{CL}$ \\
\hline 3 & TOP & $12+6$ & $47, X Y+21$ & Heart defect & POS & NEG & POS & NEG & $\mathrm{CL}$ \\
\hline 4 & TOP & $12+6$ & $46, X X$ & Achondrogenesis & POS & NEG & POS & NEG & $\mathrm{CL}$ \\
\hline 5 & TOP & $13+2$ & $46, X X$ & Myelomeningocele & POS & NEG & POS & NEG & CL \\
\hline 6 & TOP & $13+6$ & $47, X Y+21$ & Soft markers & POS & NEG & POS & NEG & $C L$ \\
\hline 7 & MIS & $11+0$ & $46, X Y$ & Normal & POS & NEG & POS & POS & $\mathrm{CH}$ \\
\hline 8 & TOP & $11+0$ & $47, X X+21$ & Soft markers & POS & NEG & POS & POS & $\mathrm{CH}$ \\
\hline 9 & TOP & $11+2$ & $47, X X+21$ & Soft markers & POS & NEG & POS & POS & $\mathrm{CH}$ \\
\hline 10 & TOP & $11+5$ & $47, X Y+21$ & Omphalocele; single umbilical artery & POS & NEG & POS & POS & $\mathrm{CH}$ \\
\hline 11 & MIS & $12+4$ & $47, X X+21$ & Soft markers & POS & NEG & POS & POS & $\mathrm{CH}$ \\
\hline 12 & TOP & $12+6$ & $45, \mathrm{X0}$ & Potter sequence type II & POS & NEG & POS & POS & $\mathrm{CH}$ \\
\hline 13 & TOP & $13+3$ & $46, X Y$ & Urinary tract malformations; hydrops & POS & NEG & POS & POS & $\mathrm{CH}$ \\
\hline 14 & TOP & $13+3$ & $47, X X+21$ & Heart defect and soft markers & POS & NEG & POS & POS & $\mathrm{CH}$ \\
\hline 15 & MIS & $13+5$ & $46, x X$ & Soft markers & POS & NEG & POS & POS & $\mathrm{CH}$ \\
\hline 16 & TOP & $13+6$ & $45, x 0$ & Potter sequence type II & POS & NEG & POS & POS & $\mathrm{CH}$ \\
\hline 17 & TOP & $13+6$ & $45, \times 0$ & Potter sequence type II & POS & NEG & POS & POS & $\mathrm{CH}$ \\
\hline 18 & TOP & $13+6$ & $46, X Y$ & Fifth digit hypoplasia; soft markers & POS & NEG & POS & POS & $\mathrm{CH}$ \\
\hline
\end{tabular}

$\mathrm{GA}=$ gestational age, $\mathrm{CL}$ and $\mathrm{CH}=$ cystic lymphangiectasia and cystic hygromas, respectively, $\mathrm{POS}=$ positive, $\mathrm{NEG}=$ negative, $\mathrm{TOP}=$ termination of pregnancy, MIS = miscarriage. 
Nuchal cystic lymphangiectasia

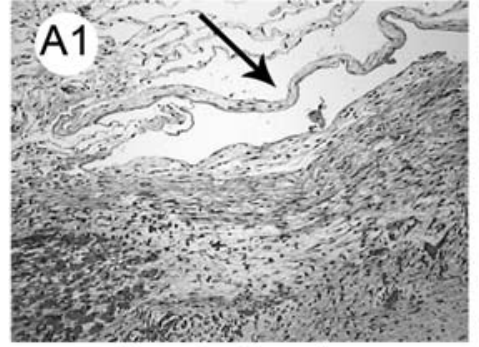

Hematoxylin eosin

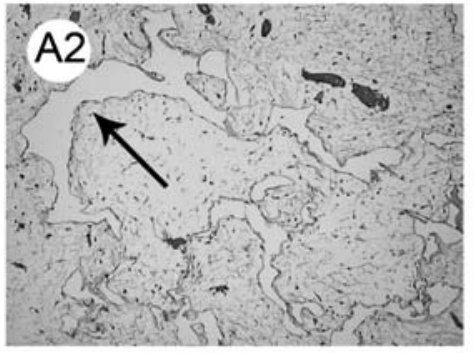

Hematoxylin eosin

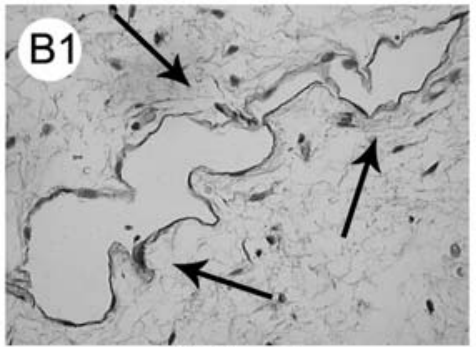

D2-40 positive

Cystic hygroma

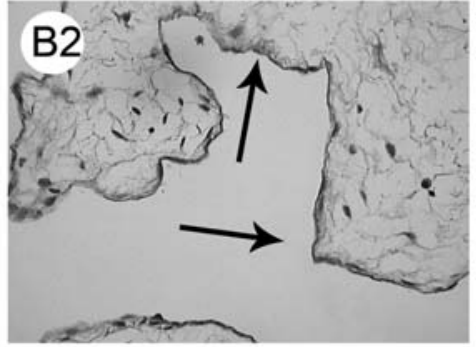

D2-40 positive

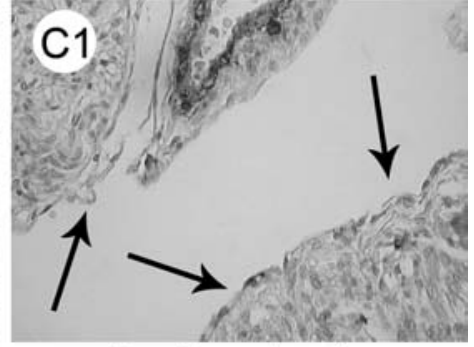

Smooth muscle actin negative

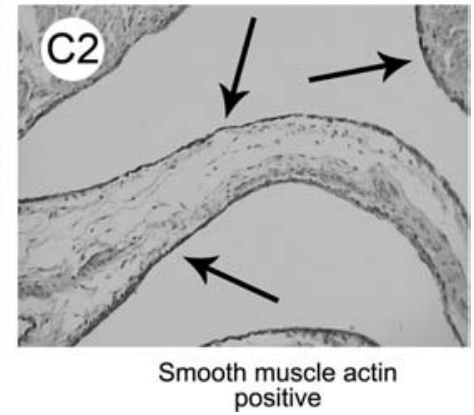

Figure 2 Panels A1, B1, and C1: case 6; gestational age 13+6; 47, XY +21 .

Panel A1 shows stretched lymphatic sacs with simple and thin walls (Hematoxylin \& Eosin staining; magnification $10 \times$ ). Panel B1 shows saccular ectasia of large collectors or lymphatic cisterns with highly positive D2-40 staining wall (magnification $10 \times$ ). Panel C1 shows thin walled lymphatic vessels with almost no smooth muscle cells (SMA staining; magnification 20X).

Panels A2, B2, and C2: case 14; gestational age $13^{+3} ; 47, \mathrm{XY}+21$.

Panel A2 is similar to Panel A1 and shows lymphatic ectasia with a thin wall (Hematoxylin \& Eosin staining; magnification $10 \times$ ). Panel B2 shows that the endothelium is homogeneous and D2-40 positive (magnification $20 \times$ ). Panel C2 shows that the lymphatic cisterns have an evident and thick wall with layers of roughly bundled SMA positive muscle fibers (magnification $20 \times$ ).

ured between the $11^{+0}$ and $13^{+6}$ weeks' gestation. The use of CD31, CD34, and D2-40 staining allowed us to conclude that the nuchal cavities are formed by lymphatic ectasia with accumulation of fluid. The demonstration that $12 / 18$ cases were SMA positive suggested the venous characteristic of the lesion of the lymphatic vessel wall due to the presence of a layer of smooth muscle cells within the lymphatic subendothelium [3]. This result is compatible with a loss of lymphatic identity. Negative SMA staining in the subendothelial lining of the remaining $6 / 18$ cases points to the presence of nuchal lymphatic ectasia, allowing to differentiate between nuchal cystic lymphangiectasia, featuring thin-walled lymphatic vessels with practically no smooth muscle cells, from cystic hygromas, consisting of large collectors or lymphatic cisterns with thick, well structured highly SMA positive walls and thus suggesting a lymphatic maldevelopmental lesion (Figure 2, panels C1 and C2). Our results (Table 1) support the reasonable hypothesis that lymphangiogenesis plays a relevant role in nuchal edema, which eventually leads to increased NT, and that increased NT is the result of a lymphatic malformation or of a developmental delay of the lymphatic system [1, 3]. The lymphatic system begins to develop from the jugular lymphatic sacs that derive from the budding of the internal jugular veins. Subsequent steps comprise the reorganization of the jugular lymphatic sacs into lymphatic nodes, which is usually completed within the first 10 weeks of gestation, and the ingrowth of the thoracic duct into the internal vein, thus connecting several lymphatic vessels. The thoracic duct is the main drainage vessel of lymph fluids [2, 3, 8].

The presence of smooth muscle cells (SMA positive) in positive CD31 and D2-40 and negative CD34 human fetuses indicates a disturbance of the venous-lymphatic differentiation. This condition points to the presence of a lymphatic dysplastic lesion such as cystic hygroma. On the other hand, the absence of smooth muscle cells, pointing to the presence of simple lymphatic ectasia without clear signs of dysplasia, is compatible with a delayed venous-lymphatic differentiation process, linked to diminished lymphatic transport and drainage that eventually leads to nuchal edema formation. Small caliber lymphatics may acquire SMA staining, thus reflecting either a developmental defect or alternatively, elevated hydrostatic pressure due to lymphatic obstruction. The delayed, but finally normal lymphatic maturation, could explain the transitory appearance of increased NT later 
than the $14^{\text {th }}$ week of gestation, which then disappears as pregnancy progresses, whereas the persistent increased NT could be linked to cystic hygroma. Thus, cystic hygroma, lymphangioma, and lymphangiectasia may represent a sort of continuum. We strongly believe that identification by ultrasound in living fetuses is not feasible. We agree with Molina et al. [13] who suggested that the clinical diagnosis of cystic hygroma should be made in the second trimester of pregnancy, thus one could distinguish between increased NT that resolves or is significantly reduced and the cystic hygroma that appears as a persistent cavity in the posterior fetal neck.

It has been reported [3] that nuchal edema in trisomy 21 and in Turner syndrome is probably caused by different mechanisms. In that paper, the authors observed the absence of the jugular lymphatic sac and lymphatic hypoplasia in Turner syndrome, whereas in trisomy 21 they observed nuchal skin lymphatic vessel ectasia. Thus, these data could indicate disrupted lymphatic endothelial cell (LEC) differentiation in trisomy 21 and a lack of LEC differentiation in Turner syndrome. Our study did not allow us to correlate between various IHC patterns and karyotype abnormalities (Table 1).

To date, few, conflicting, and inconclusive reports are available on this topic $[3,5,7]$. Measuring NT is carried out for a long time, and although several attempts were made to explain the origin of increased NT in aneuploidy, structural anomalies, as well as a great deal of genetic syndromes, no definitive conclusion could be reached. Delayed or, in turn, disturbed fetal lymphatic system development, both of which are expressions of a common developmental process, is a promising field of research. Our study seems to provide further positive clues in this direction. We suggest that the number of postmortem examinations performed on abortuses with increased NT should be increased as should be the recruitment of fetuses older than 14 weeks of gestational age in whom increased NT eventually resolved.

\section{References}

[1] Bekker MN, Haak MC, Rekoert-Hollander M, Twisk J, Van Vugt JM. Increased nuchal translucency and distended jugular lymphatic sacs on first-trimester ultrasound. Ultrasound Obstet Gynecol. 2005;25:239-45.

[2] Bekker MN, van den Akker NM, Bartelings MM, Arkesteijn JB, Fischer SG, Polman JA, et al. Nuchal edema and venous-lymphatic phenotype disturbance in human fetuses and mouse embryos with aneuploidy. J Soc Gynecol Investig. 2006;13:209-16.

[3] Bekker MN, van den Akker NMS, de Mooij YM, Bartelings MM, van Vugt JMG, Gittenberger-de Groot AC. Jugular lymphatic maldevelopment in Turner Syndrome and Trisomy 21: different anomalies leading to nuchal edema. Reproductive Sciences. 2008;15:295-304.

[4] Carvalho JS. The fetal heart or the lymphatic system or...? The quest for the etiology of increased nuchal translucency. Ultrasound Obstet Gynecol. 2005;25:215-20.

[5] Chitayat D, Kalousek DK, Bamforth JS. Lymphatic abnor- malities in fetuses with posterior cervical cystic hygroma. Am J Med Genet. 1989;33:352-6.

[6] Dabbs DJ. Diagnostic immunohistochemistry. 2nd ed. Churchill Livingstone: Philadelphia; 2006.

[7] Gittenberger-De Groot AC, Van Den Akker NM, Bartelings MM, Webb S, Van Vugt JM, Haak MC. Abnormal lymphatic development in trisomy 16 mouse embryos precedes nuchal edema. Dev Dyn. 2004;230:378-84.

[8] Haak MC, Bartelings MM, Jackson DG, Webb S, van Vugt JM, Gittenberger-de Groot AC. Increased nuchal translucency is associated with jugular lymphatic distension. Hum Reprod. 2002;17:1086-92.

[9] Haak MC, Bartelings MM, Gittenberger-De Groot AC, Van Vugt JM. Cardiac malformations in first-trimester fetuses with increased nuchal translucency: ultrasound diagnosis and postmortem morphology. Ultrasound Obstet Gynecol. 2002;20:14-21.

[10] Kahn HJ, Marks A. A new monoclonal antibody, D2-40, for detection of lymphatic invasion in primary tumors. Lab Invest. 2002;82:1255-7.

[11] Knisely AS. The pathologist and the hydropic placenta, fetus, or infant. Semin Perinatol. 1995;19:525-31.

[12] L'Herminé-Coulomb A. Increased nuchal translucency pathophysiology. J Gynecol Obstet Biol Reprod (Paris). 2005;34(1 Suppl):S89-96.

[13] Molina FS, Avgidou K, Kagan KO, Poggi S, Nicolaides KH. Cystic hygromas, nuchal edema, and nuchal translucency at 11-14 weeks of gestation. Obstet Gynecol. 2006;107: 678-83.

[14] Malone FD, Canick JA, Ball RH, Nyberg DA, Comstock $\mathrm{CH}$, Bukowski R, et al. First-trimester or second-trimester screening, or both, for Down's syndrome. N Engl J Med. 2005;353:2001-11.

[15] Pajkrt E, de Graaf IM, Mol BW, van Lith JM, Bleker OP, Bilardo CM. Weekly nuchal translucency measurements in normal fetuses. Obstet Gynecol. 1998;91:208-11.

[16] Sauter B, Foedinger D, Sterniczki B, Wolff K, Rappersberger K. Immunoelectron microscopic characterization of human dermal lymphatic microvascular endothelial cells. Differential expression of CD31, CD34 and type IV collagen with lymphatic endothelial cells vs. blood capillary endothelial cells in normal human skin, lymphangioma, and hemangioma in situ. J Histochem Cytochem. 1998:46; 165-76.

[17] Schacht V, Dadras SS, Johnson LA, Jackson DG, Hong YK, Detmar M. Up-regulation of the lymphatic marker podoplanin, a mucin-type transmembrane glycoprotein, in human squamous cell carcinomas and germ cell tumors. Am J Pathol. 2005;166:913-21.

[18] Snijders RJ, Noble P, Sebire N, Souka A, Nicolaides KH. UK multicentre project on assessment of risk of trisomy 21 by maternal age and fetal nuchal-translucency thickness at 10-14 weeks of gestation. Fetal Medicine Foundation First Trimester Screening Group. Lancet. 1998;352: 343-6.

[19] Souka AP, Krampl E, Bakalis S, Heath V, Nicolaides KH. Outcome of pregnancy in chromosomally normal fetuses with increased nuchal translucency in the first trimester. Ultrasound Obstet Gynecol. 2001;18:9-17.

The authors stated that there are no conflicts of interest regarding the publication of this article.

Received February 17, 2009. Revised April 30, 2009. Accepted May 4, 2009. Previously published online July 10, 2009. 